\title{
Shape Degeneration and Multi-object Search Basing on Multi-shape Priors
}

\author{
Song Chunhe* \\ College of Information Science and Technology, Northeastern University \\ Shenyang, 110819, China \\ tedachun@163.com \\ Zhao Hai \\ College of Information Science and Technology, Northeastern University \\ Shenyang, 110819, China \\ zhhai@mail.neuera.com \\ Jing Wei \\ College of Information Science and Technology, Northeastern University \\ Shenyang, 110819, China \\ neujw@126.com \\ Zhu Hongbo \\ College of Software, Northeastern University \\ Shenyang, 110819, China \\ zhuhb@mail.neuera.com
}

\begin{abstract}
This paper proposed a framework of segmenting multiple targets basing on multiple shape priors. The key novel idea in the proposed framework is the shape degeneration model. By breaking the balance between the competition regions adaptively, the proposed model can degenerate the region which is with low similarity with any of the shape priors, until this region completely disappears. Meanwhile the proposed model can also merge the regions that belong to a single object automatically, which is especially important when some of objects are in partial occlusion. Additionally, we presented two new models in the proposed framework. One is a new local model of active contour, which can segment image with intensity inhomogeneity correctly. The other is a generalized shape prior model, which extends the intrinsic alignment model, and can take extra affine parameters into account in the alignment process of shape priors. Experimental results show the validity of the proposed method.
\end{abstract}

Keywords: image segmentation, level set, shape priors, multi-object segmentation.

\section{Introduction}

Segmentation is a key issue of imaging and vision, which aims at separating the image domain into regions with consistent properties. A vast amount of researches

*Mailbox 377, Northeastern University, Shenyang, 110819, China. were performed during the past several decades toward complete automated solutions for general purpose of image segmentation. Variational techniques [2], statistical methods [12-15], combined approaches [6-11], curve-propagation techniques [1], and methods that perform non-parametric clustering [17-19] are some 
examples. In recent years the level set method has become a popular framework for image segmentation, which has been adapted to segment images based on numerous low-level criteria such as edge consistency, intensity homogeneity, texture information and motion information. More recently, it was proposed to integrate prior knowledge about the shape of expected objects into the level set framework $[3-5,10,14]$.

\subsection{Related works}

In [14], Ballerini et al. suggested to represent a set of training shapes by their signed distance function sampled on a regular grid and to apply principal component analysis to this set of training vectors. Subsequently they enhanced a geodesic active contours segmentation process by adding a term to the evolution equation which draws the level set function toward the function which is most probable according to the learnt distribution. Rousson and Paragios [2] performed PCA to obtain a set of eigenvectors and subsequently reformulated the segmentation process to directly optimize the parameters associated with the first few deformation modes. Chan and Zhu [3] proposed to impose prior knowledge onto the segmenting contour extracted after each generation of the level set function. While this approach allows introducing shape information into the segmentation process, it is not entirely in the spirit of the level set scheme since the shape prior acts on the contour and is therefore not capable of modeling topological changes. Fussenegger et al. [4] and Dambreville et al. [5] imposed shape information into the variation formulation of the level set scheme, either by a model of local Gaussian fluctuations around a mean level set function or by global deformation modes along the lines of [7]. More recently, level set formulations were proposed to allow imposing dynamical shape priors and concepts of tracking, to apply shape knowledge selectively in certain image regions, or to impose multiple competing shape priors so as to simultaneously reconstruct several independent objects in a given image sequence [10].

\subsection{Main issues}

Basing on a suitable model without shape priors, one can get the result of unsupervised segmentation. And if introducing shape priors into the segmentation process, regions with the contour similar to one of the shape priors can be separated from the background. As shown

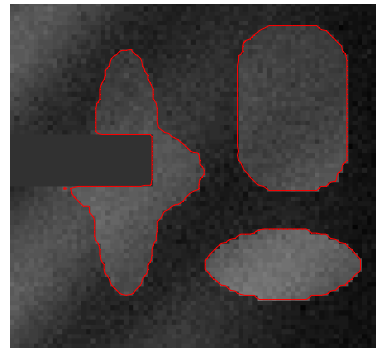

(a)

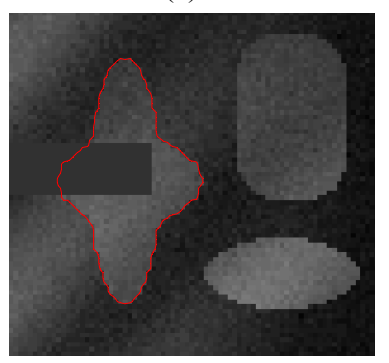

(c)

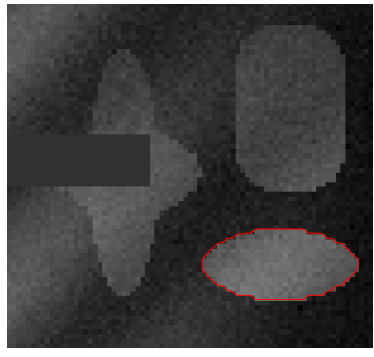

(b)

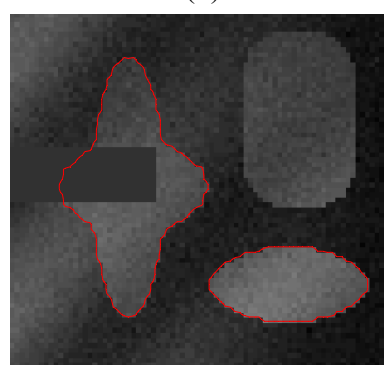

(d)
Fig. 1. Segmentation results: (a): segmentation without prior; (b) and (c): segmentation with priors; (d): segmentation basing on the proposed framework

in Fig.1, (a) is the segmentation result without shape priors, while (b) and (c) are the results with one special shape prior. Noticing that if two or more shape priors are used in this process, the result will be either (b) or (c), which is mainly determined by the initial position of the zero level set function. However, no matter with or without shape priors, it is hard to get the segmentation result just like Fig.1(d), which includes more than one region with the similar contour to the shape priors.

Obviously the result of Fig.1(d) is very important, as there may be more than one region of interests (ROIs) in the image. And the main task of this paper is to present a framework to get multiple ROIs basing on multiple shape priors using level set method.

\subsection{Contributions}

This paper proposed a framework to segment multiple ROIs basing on multiple shape priors using level set method, which mainly consists of four steps: in the first step, a new local level set method is proposed to segment objects in the image. Then in the second step, the connected components labeling algorithm is used to tag the main regions of the results from step 1. In the third step, for each of the tagged region, a level set function will be generated, and then a novel shape degeneration model is proposed to exclude regions with 
the contour dissimilar with any of shape priors. Finally in the last step, all level set method will be combined to get the multiple ROIs.

A key idea of the active contour is the region competition, which means that the contour is driven by the region forces between the inner and outer of the contour until balance. While the proposed shape degeneration in this paper bases on an inverse idea. By breaking the balance between competing regions adaptively, regions which are with low similarity with any of shape priors will be generated, until completely disappear. Meanwhile the proposed model will merge the regions that belong to one single object automatically, which is especially important when some of objects are in partial occlusion.

There are two additional new models presented in the proposed framework. The first is a new local model of active contour. It has been found that kernel active contour can yield better results on the image with intensity inhomogeneity. In [7], the kernel is a Gaussian with a scale parameter $\sigma$, while in [8] the kernel is a $0-1$ characteristic function in term of a radius parameter $r$. As discussed in [7], a larger size kernel will yield a more stable result, while a smaller size kernel will yield a more accurate result but may stop in a local minimum. In this paper, we proposed a new local active contour with adaptive kernel, from the experimental results it can be seen that the proposed model can get more accurate and stable results than $[7,8]$. The second is a general shape prior model. In [10], Cremers et al. proposed to use invariance moments to deal with the relative translation and scale between the shape priors and the objects in the image. In this paper, we extended the Cremers et al.'s idea and proposed a general intrinsic alignment model. This model can take extra affine parameters into account, with which the similarity between current contour and shape prior can be get easily.

\section{Level Set Based Segmentation}

Originally introduced in the community of computational physics as a means of propagating interfaces [1], the level set method has become a popular framework for image segmentation. The central idea is to implicitly represent a contour $C$ in the image plane $\Omega$ as the zero-level of an embedding function $\phi$ :

$$
C=\{x \in \Omega \mid \phi(x)=0\}
$$

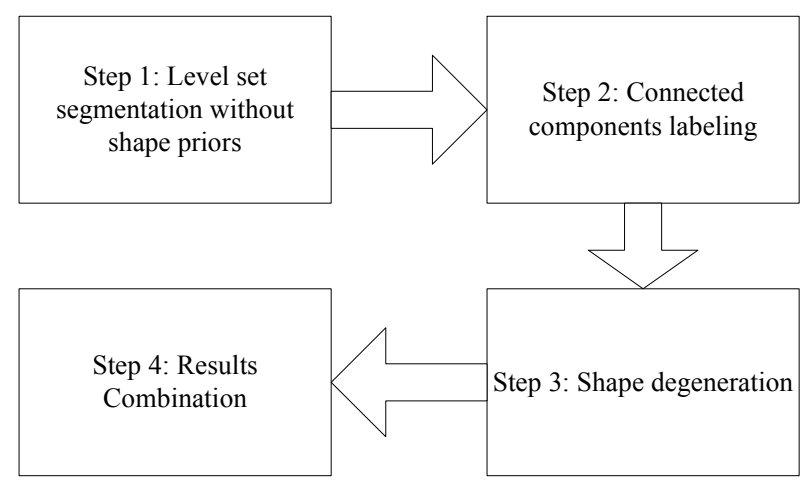

Fig. 2. The proposed framework

Rather than directly evolving the contour $C$, one can evolve the level set function $\phi$. The two main advantages are that firstly it does not need to deal with control or mark points. And secondly, the embedded contour is free to undergo topological changes such as splitting and merging which makes is well-suited for the segmentation of multiple or multiply-connected objects. Mumford and Shah first proposed to minimize the following formula (Mumford-Shah, MS) to carry out image segmentation [2]:

$$
\begin{aligned}
F^{M S}(u, C)= & \int_{\Omega}(u-I)^{2} d x \\
& +\mu \int_{\Omega \backslash C}|\nabla u|^{2} d x+v|C|
\end{aligned}
$$

where $I$ is the image, $|C|$ is the length of the contour. $u$ is the approximate image of $I$ and smooth element of each link segmented by contour $C$. In fact it is very difficult to minimize the MS formula, because the contour is unknown, and the function $F^{M S}(u, C)$ is non-convex. In order to address these issues, T. Chan and L. Vese proposed piecewise constant model (PC) and define the following function:

$$
\begin{aligned}
& F^{C V}\left(C, c_{1}, c_{2}\right)=\mu \cdot|C| \\
& \quad+\lambda_{1} \int_{\text {outside }(c)}\left|I(x)-c_{1}\right|^{2} d x \\
& \quad+\lambda_{2} \int_{\text {outside }(c)}\left|I(x)-c_{2}\right|^{2} d x
\end{aligned}
$$

where $c_{1}$ and $c_{2}$ are the mean densities of inside and outside of the contour. The optimal constants $c_{1}$ and $c_{2}$ that minimize the above energy are the averages of the intensities in the entire regions outside and inside of $C$. Such optimal constants $c_{1}$ and $c_{2}$ can be far away from the original image data if the intensities within outside or inside of $C$ are not homogeneous. They do not contain any local intensity information, 
which is crucial for segmentation of images with intensity inhomogeneity. As a result, the PC model generally fails to segment images with intensity inhomogeneity. Later Li et al. [7] and Lankton et al. [8] proposed local active contour models, respectively, to address the issue. They used the kernel function to each pixel to collect local information around it. The only difference between the two models is the kernel function. In [7], a Gauss kernel was used while in [8] a characteristic function in term of a radius parameter was used.

\section{Multi-ROIs Searching Basing on Multiple Shape Priors}

The framework of the proposed method in this paper is shown in Fig.2, which includes four steps: level set evolution without shape priors, connected components labeling, shape degeneration, and results combination. In the rest of section we will discuss the process of the proposed framework step by step.

\subsection{A new local model of active contour}

A good energy formulation is the base of the entire segmentation work. In the first step of the proposed framework, segmentation is carried out without shape priors and the corresponding level set energy is shown as follows:

$$
E=E_{\text {region }}+\lambda_{1}|C|+\lambda_{2} E_{\text {regulation }}
$$

The formula contains three items: $E_{\text {region }}$ is the energy of the image, $|C|$ is the length of zero level set and $E_{\text {regulation }}$ is the regulation item [6] which is used to overcome the re-initiation issue. We simply give the formulas of $|C|$ and $E_{\text {regulation }}$ as follows:

$$
\begin{aligned}
& |C|=\int_{\Omega}\left|\nabla H_{\varepsilon}(\phi(x, y))\right| d x d y \\
& E_{\text {regulation }}=\int \frac{1}{2}(|\nabla \phi(x)|-1)^{2} d x
\end{aligned}
$$

where $\nabla$ is the gradient, and $H_{\varepsilon}$ is the smoothedHeaviside function:

$$
H_{\varepsilon}=\frac{1}{2}\left[1+\frac{2}{\pi} \arctan \left(\frac{x}{\varepsilon}\right)\right]
$$

$\varepsilon$ is a positive number.

Another key point in Eq.(4) is $E_{\text {region }}$, which is the energy of the image. Firstly it is a local energy:

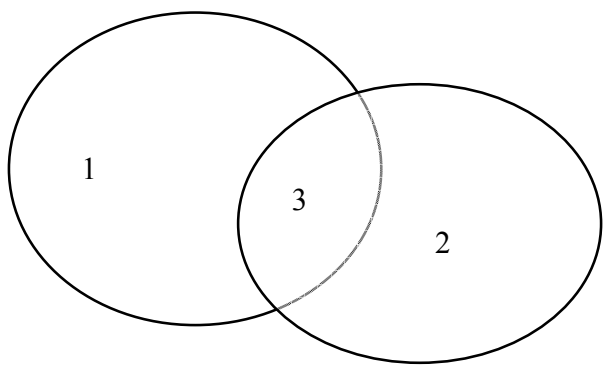

Fig. 3. The definition of the stability

$$
\begin{gathered}
E_{\text {region }}=\lambda_{3} \int_{\Omega} K H(\phi)\left(I(x)-c_{1}\right)^{2} d x \\
+\int_{\Omega} K(1-H(\phi))\left(I(x)-c_{2}\right)^{2} d x
\end{gathered}
$$

where $c_{1}$ and $c_{2}$ are as follows:

$$
c_{1}(\phi)=\frac{\int_{\Omega} I(x, y) K H(\phi(t, x, y)) d x d y}{\int_{\Omega} K H(\phi(t, x, y)) d x d y}
$$

$$
c_{2}(\phi)=\frac{\int_{\Omega} I(x, y) K(1-H(\phi(t, x, y))) d x d y}{\int_{\Omega} K(1-H(\phi(t, x, y))) d x d y}
$$

In [7], the $\mathrm{K}$ is a Gaussian kernel with a scale parameter $\sigma$, and in [8], the $\mathrm{K}$ is a $0-1$ characteristic function in term of a radius parameter $r$. Different with the above kernels, in this paper, we used the kernel shown as follows:

$$
K\left(P^{0}, P^{1}\right)= \begin{cases} & \left\|P_{x}^{0}-P_{x}^{1}\right\|<\gamma \\ & \&\left\|P_{x}^{0}-P_{x}^{1}\right\|<\gamma \\ 0, & \text { otherwise }\end{cases}
$$

where $P^{0}$ is the current point, $P_{x}^{0}$ is the x-coordinate $P^{0}$. $P^{1}$ is a neighbor of $P^{0}, d$ is a positive constant, as discussed in [7][8], a larger size kernel will yield a more stable result, while a smaller size kernel will yield a more accurate result but may stop in a local minimum. As we want to segment the object from the background, and it is reasonable to believe that the size of the kernel is related to the object's size, in this paper, we choose:

$$
\gamma=\sqrt{\int_{\Omega} H(\phi(x, y)) d x d y}
$$

The kernel used in this paper can be considered as the kernel in the [7] with unique weight or the kernel in the [8] with square shape. The advantage of Eq.(11) over 


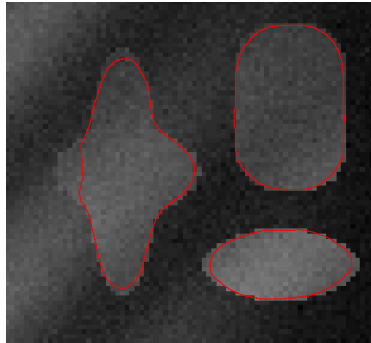

(a)

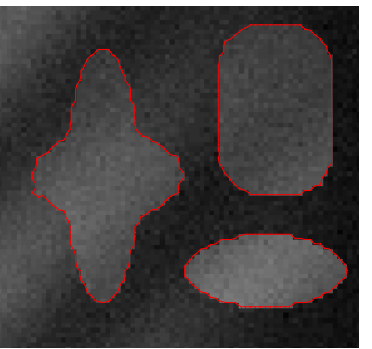

(b)

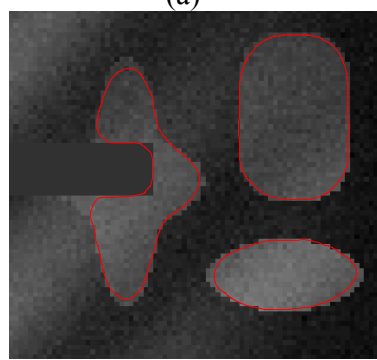

(c)

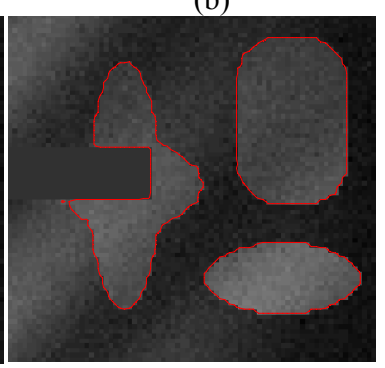

(d)
Fig. 4. Segmentation results: (a): CV model, (b): the proposed model without priors, (c): result of [7], (d): the proposed model without priors.

that in [7] and [8] is that it is with lower computation consumption and more convenient to implement in the level set evolution.

Only when the curve is nearly stable then the step 1 is finished. Now we define the stability of the curve using Eq.(13):

$$
\eta=1-\frac{\int_{\Omega}\left(H\left(\phi_{j+\alpha}\right)-H\left(\phi_{j}\right)\right)^{2} d x}{\int_{\Omega}\left(H\left(\phi_{j+\alpha}\right)+H\left(\phi_{j}\right)\right)^{2} d x}>\tau
$$

where $\alpha$ and $\tau$ are the positive numbers, $\phi_{j}$ and $\phi_{j+\alpha}$ are the $j$-th and $(j+\alpha)$-th generation of the level set function.

The meaning of Eq.(13) can be seen in Fig.3. Supposing region $1 \cup$ region 3 is $H\left(\phi_{j}\right)$, and region $2 \cup$ region 3 is $H\left(\phi_{j+\Delta}\right)$. It is easy to find that $\int_{\Omega}\left(H\left(\phi_{j+\Delta}\right)-H\left(\phi_{j}\right)\right)^{2} d x=$ region $1 \cup$ region 2 , and $\int_{\Omega}\left(H\left(\phi_{j+\Delta}\right)+H\left(\phi_{j}\right)\right)^{2} d x=$ region $1 \cup$ region $2 \cup$ region 3. If the difference between $H\left(\phi_{j}\right)$ and $H\left(\phi_{j+\Delta}\right)$ is close to 0 , then $\int_{\Omega}\left(H\left(\phi_{j+\Delta}\right)-H\left(\phi_{j}\right)\right)^{2} d x$ will be close to 0 , and the same to $\eta$. If there is no shared region between $H\left(\phi_{j}\right)$ and $H\left(\phi_{j+\Delta}\right)$, then $\eta$ will be 1 . So $\eta$ can be used to represent the similarity between $H\left(\phi_{j}\right)$ and

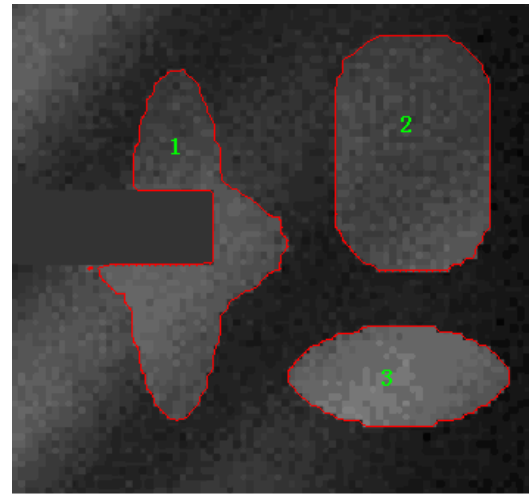

Fig. 5. Connected components labeling

$H\left(\phi_{j+\Delta}\right)$, in other words, the stability of the evolving curve.

A example of the results of the proposed local active contour is shown in Fig.4, in which (a) is the result of $\mathrm{CV}$ model, (c) is the result of [7], (b) and (d) are the results of the proposed model. From Fig.4(b) it can be seen that our model can segment image with intensity inhomogeneity correctly, while $\mathrm{CV}$ model yields a wrong result. From Fig.4(c) and Fig.4(d) it can be seen that, as using an adaptive kernel size, our model can get a more accurate result than that of [7] when there are some sharp corners in the image.

\subsection{Connected components labeling}

After the segmentation process without shape priors, there will be several disconnected regions which need to be tagged. There exists several components labeling algorithm, and as it is not the key issue of the paper, we simply use the method proposed by He et al. [20]. But different from general labeling method, in the proposed framework, only the regions with the area bigger than a predefined threshold can be tagged. A sample of labeling result is shown in Fig.5. In this experiment the threshold is 5. Noticing that there is an unlabeled region near the region 1 , as the area of the unlabeled region is smaller than the predefined threshold. After the labeling step, each tagged region generates a level set function as Eq.(14):

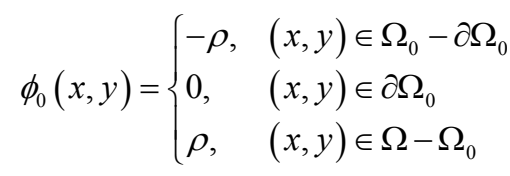

where $\rho$ is a positive number. 


\subsection{Shape degeneration}

\subsubsection{Shape energy}

The core work of the step 3 is to degenerate regions with contour dissimilar to any of shape priors and deal with the situation that more than one region belong to a single objects, which is usually in occlusion. In order to introduce shape priors into level set, a novel shape energy $E_{\text {shape }}$ is proposed, which is the sum of weighted kernel density estimation of the difference between the current shape and shape priors. $E_{\text {shape }}$ can be written as Eq.(15):

$$
E_{\text {shape }}=\frac{\sum_{i=1}^{N} \alpha_{i} d\left(H(\phi), H\left(\phi_{i}\right)\right)}{2 \sigma^{2} \sum_{i=1}^{N} \alpha_{i}}
$$

where $d\left(H(\phi), H\left(\phi_{i}\right)\right)$ is the distance between $H(\phi)$ and $H\left(\phi_{i}\right)$ in the kernel space, $\mathrm{N}$ is the number of shape priors, and $\alpha_{i}$ is the shown as follows:

$$
\alpha_{i}=\exp \left(-\frac{1}{2 \sigma^{2}} d\left(H(\phi), H\left(\phi_{i}\right)\right)\right)
$$

In [10], it considered only the translation and scale which were calculated by the invariance moments. In this paper, we extend the idea of [10] and take the rotation into account. The translation $T$, scale $S$ and rotation angle $A$ are calculated as follows:

$$
\begin{gathered}
S=\sum_{x} \sum_{y} H(\phi(x, y)) \\
T\left(x_{c}, y_{c}\right)=\left(\frac{m_{10}}{S}, \frac{m_{01}}{S}\right) \\
A=\arctan \left(\frac{b}{a-c}\right) / 2
\end{gathered}
$$

where:

$$
\begin{aligned}
& m_{10}=\sum_{x} \sum_{y} x H(\phi(x, y)), m_{01}=\sum_{x} \sum_{y} y H(\phi(x, y)), \\
& m_{20}=\sum_{x} \sum_{y} x^{2} H(\phi(x, y)), m_{02}=\sum_{x} \sum_{y} y^{2} H(\phi(x, y)), \\
& m_{11}=\sum_{x} \sum_{y} x y H(\phi(x, y)), a=\frac{m_{20}}{S}-x_{c}^{2}, \\
& b=2\left(\frac{m_{11}}{S}-x_{c} y_{c}\right) \text { and } c=\frac{m_{02}}{S}-y_{c}^{2} .
\end{aligned}
$$

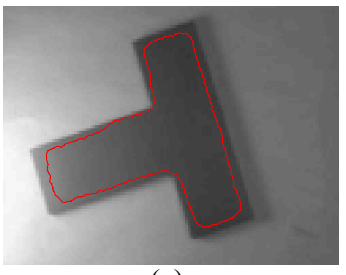

(a)

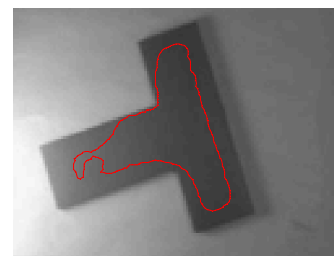

(c)

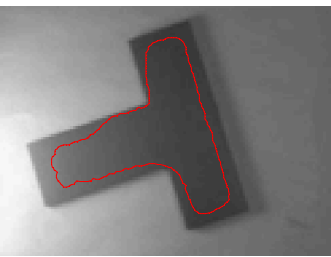

(b)

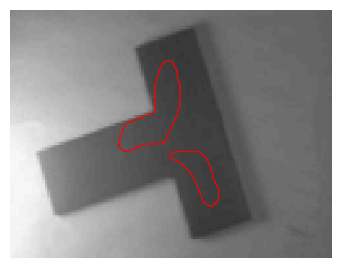

(d)
Fig. 6. An example of the shape degeneration process

Take $T$ as an example, we firstly calculate $T$ using Eq.(18) for each shape prior and record them. Then in each generation of the level set function, we calculate $T$ for the zero level set function which is denoted by $T_{j}^{*}$ for the $\mathrm{j}$-th generation, then instead of calculating $d\left(H(\phi), H\left(\phi_{i}\right)\right)$ in Eq.(16), we calculated $T_{j}^{*}, T_{i}$ and $d_{j}\left(H\left(\phi-T_{j}^{*}\right), H\left(\phi_{i}-T_{i}\right)\right)$ using Eq.(17) and Eq.(18), which means to move the center of zero level set $\phi$ and shape priors to the origin of the coordinate. The operations are the same for scale $S$ and rotation angle $A$.

Now the remaining problem is how to choose $\sigma$, in this paper, we dynamically set $\sigma$ to be the mean distance between current contour and the shape prior:

$$
\sigma^{2}=\frac{1}{N} \sum_{i=1}^{N} d\left(H(\phi), H\left(\phi_{i}\right)\right)
$$

The idea of Eq.(20) is to split the differences between the current shape with shape priors.

\subsubsection{Shape Degeneration}

In order to degenerate the region with the shape dissimilar to any of shape priors, we can break the balance of region competition which can be considered as the reverse process of $\mathrm{CV}$ and some improved models [7,8]. To achieve this, the $\lambda_{3}$ in Eq.(4) is replaced by $\varphi$ as shown in Eq.(21). It is very important for the degeneration process. We also firstly give the formula of $\varphi$. 


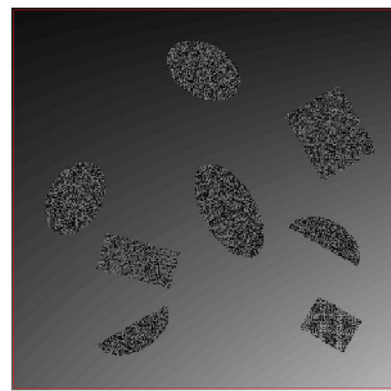

(a)

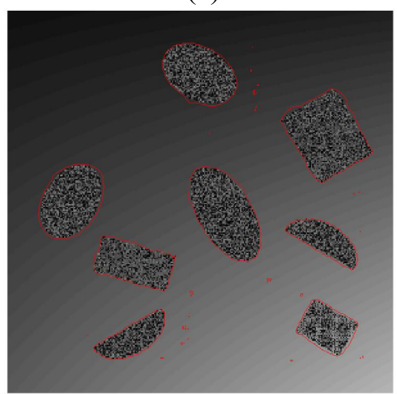

(e)

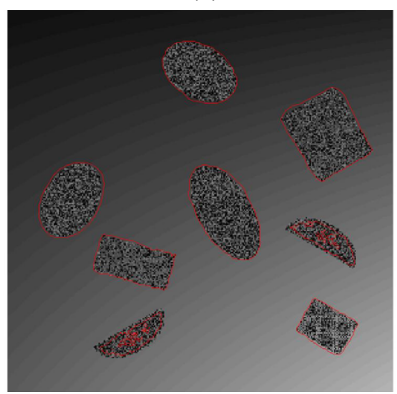

(i)

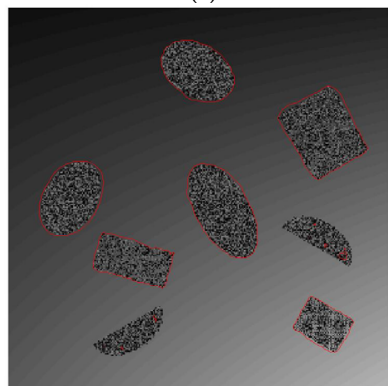

(m)

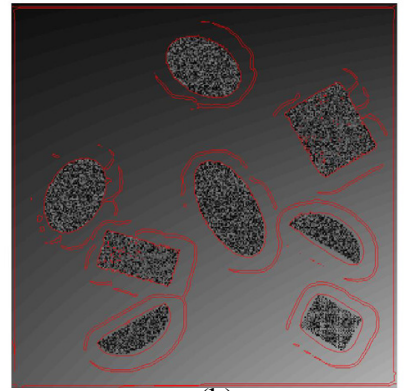

(b)

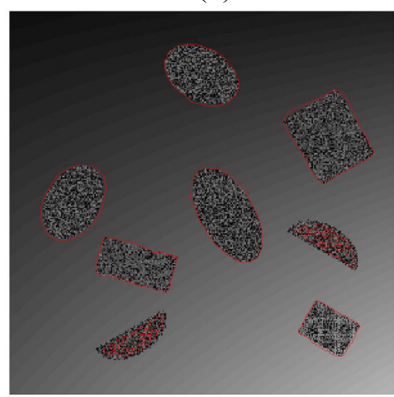

(f)

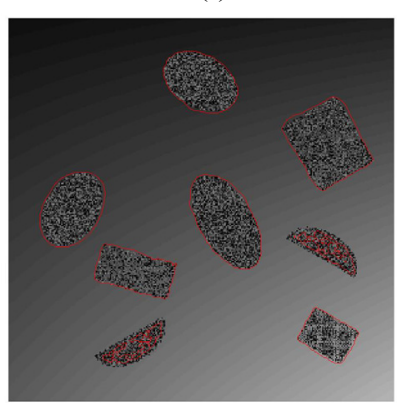

(i)

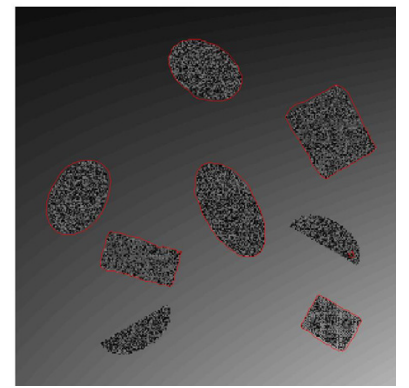

(n)

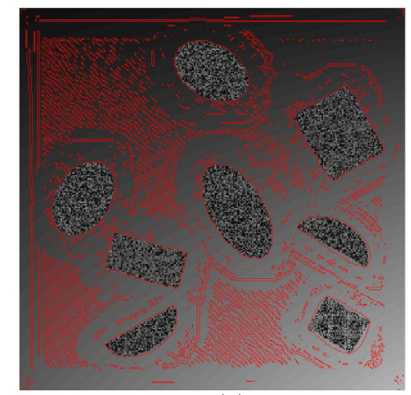

(c)

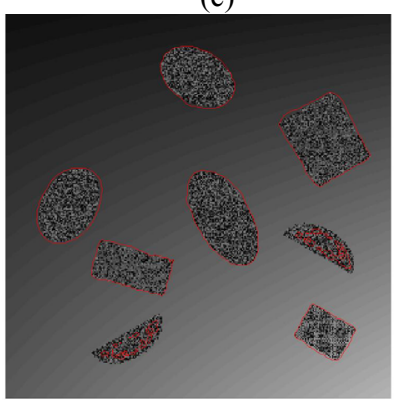

(g)

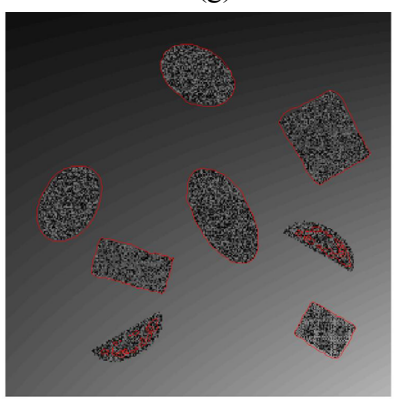

(k)

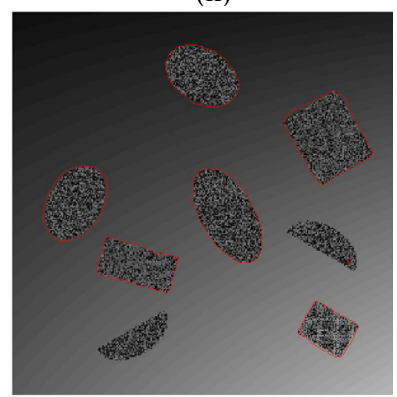

(o)

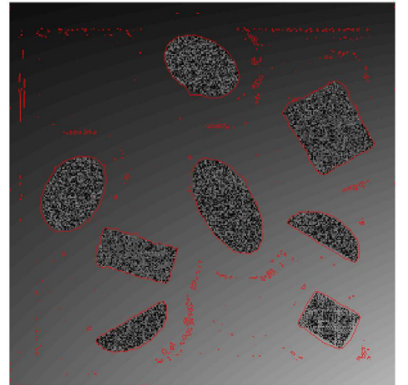

(d)

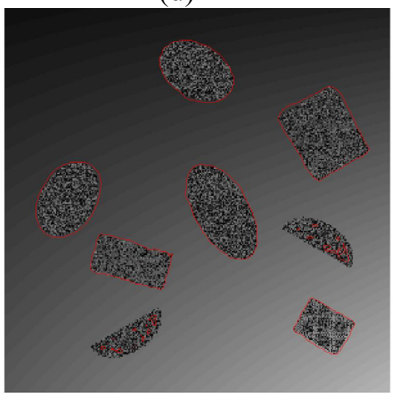

(h)

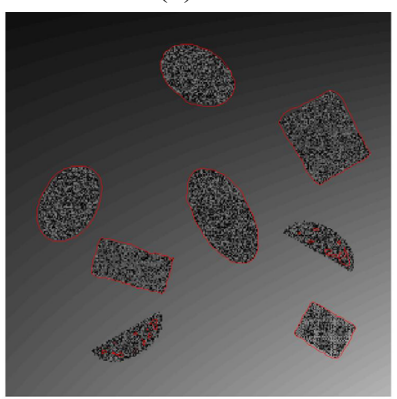

(1)

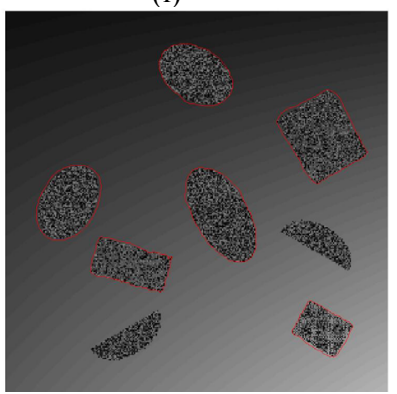

(p)

Fig. 7. An example of the shape degeneration process

$$
\varphi=\lambda_{4} \exp \left(-\frac{E_{\text {shape }}}{\lambda_{5}}\right)
$$

where $\lambda_{4}$ and $\lambda_{5}$ are two positive numbers.

From Eq.(21) it can be seen that $\varphi$ is related to $E_{\text {shape }}$ and in the range of 0 and $\lambda_{4}$.
When $E_{\text {shape }}$ increases, the value of $\varphi$ will decrease, and vice versa. Fig.6 gives an example of shape degeneration process.

According to region competition theory, if the outer's push force is bigger than the inner's, the curve will move to inner. When the stability of the level set function is bigger than $\tau$, then the contour will be 
compared to shape priors. Noticing that the contour and all of shape priors will be regularized according Eq.(17) - Eq.(19). If the curve's contour is similar to one of the shape prior, then the in Eq.(21) will be close to 1 and the level set function will evolve guided by the shape prior as shown in the Fig.1(c) and Fig.1 (d). But if the region's contour is dissimilar to any of the shape prior, then the in Eq.(21) will be close to 0, will make the inner push force decrease immediately, then the contour will shrink until completely disappear, just like the process from Fig.6(a) to Fig.6(d). One except case is that the shrinking curve becomes similarly to one of the shape prior again accidentally. But as the basic idea of the model is region competition, if the region can keep alive, it must find a suitable location in the image area where both fits the region competition and the shape prior, or else it will disappear after several number of evolution steps.

\subsection{Results combination}

The last step is rather simple. Supposing the final level set of each region are donated by $\left\{\phi^{j}\right\}_{j=1}^{m}$, where $\mathrm{m}$ is the number of tagged regions. Then in this step, we only need to do:

$$
\phi_{f}=H\left(\sum_{j=1}^{m} \phi^{j}\right)
$$

where $\phi_{f}$ is the final result of level set.

When the object is in in partial occlusion and one or more parts of it are labeled, all of regions will evolve independently. If some of them survive and successfully segment the object correctly supervised by shape priors, in the step of results combination, they will be with the same zero level set function, that is why our method can merge the regions belong to a single object when it is in partial occlusion.

\section{Experiments}

In this section we will carry out a more complex but intuitionistic experiment. The basic image is synthetical and shown in Fig.7(a). The purpose is to segment several regions with the shape of rectangle and ellipse. The related parameter: $\lambda_{1}=0.002 * 255 * 255, \lambda_{2}=1$, $\rho=2, \varepsilon=0.1, \lambda_{4}=0.5, \lambda_{5}=1, \tau=0.95$.

As shown in Fig.7(a). There are two rectangles, two ellipses and two half of ellipses in the image with intensity inhomogeneity. From Fig.7(a) to Fig.7(e) is the first step of the proposed algorithm. In Fig.7(e), regions with area bigger than 5 are labeled. The rests in Fig.(7) are the process of shape degeneration. It can be seen that the proposed framework can get the segmentation result with more than one shape priors, which is hard for other shape priors based segmentation process.

\section{Conclusion and Future Works}

Using a suitable model without shape priors, one can get the unsupervised segmentation results. And if introducing shape priors into the segmentation process, the object with the contour similar to the shape priors can be segmented. However, no matter with or without shape priors, it is hard to get the segmentation result with more than one shape priors. This paper proposed a framework of multi-object search basing on multi-shape priors. With this framework, one can get more than one object in the segmented regions whose contour is similar to some of the shape priors. However there are still some issues to be solved. For example, the proposed framework is made up of two independent steps which are connected by the componets labeling step, and the stability of the level set is defined to determine when the first step is stopped. So how to integrate the two steps into a single mathematic framework is our next work.

\section{Acknowledgements}

This work was supported in part by the National Natural Science Foundation of China (50877004), and the Cultivation Fund of the Key Scientific and Technical Innovation Project Ministry of Education of China (NO708026).

The authors would like to thank the anonymous reviewers for the constructive comments which greatly improve the quality of the paper.

\section{References}

1. Osher, S.J. and Sethian, J.A. 1988. Fronts propagation with curvature dependent speed: Algorithms based on Hamilton-Jacobi formulations. J. of Comp. Phys., 79:1249.

2. Rousson, M. and Paragios, N. 2002. Shape priors for level set representations. In A. Heyden et al., editors, Europ. Conf. on Comp. Vis., volume 2351 of Lect. Not. Comp. Sci., pages 78-92. Springer.

3. Chan, T. and Zhu, W. 2003. Level set based shape prior segmentation. Technical Report 03-66, Computational Applied Mathematics, UCLA, Los Angeles. 
4. Michael Fussenegger, Peter M. Roth, Horst Bischof and Axel Pinz, On-Line, Incremental Learning of a Robust Active Shape Model, DAGM2006, LNCS4174, pp. 122131, 2006.

5. Dambreville S, Rathi Y, Tannenbaum A,IEEE A Framework for Image Segmentation Using Shape Models and Kernel Space Shape Priors, PAMI , 2008, 30(8):1385-1399.

6. Chan, T.F. and Vese, L.A. 2001. Active contours without edges. IEEE Trans. Image Processing, 10(2):266-277.

7. Chunming Li Chiu-Yen Kao Gore, J.C. Zhaohua Ding, Minimization of Region-Scalable Fitting Energy for Image Segmentation, IEEE Trans. Image Processing, 2008, 17(10): 1940-1949.

8. Lankton S, Tannenbaum A, Localizing Region-Based Active Contours, IEEE Trans. Image Processing, 2008, 17(11):2029-2039

9. Chunming Li, Chenyang $\mathrm{Xu}$, Changfeng Gui, and Martin D. Fox 1, Level Set Evolution Without Reinitialization: A New Variational Formulation, CVPR, 2005.

10. Cremers, D., Osher, S. J., \& Soatto, S.. Kernel density estimation and intrinsic alignment for shape priors in level set segmentation. International Journal of Computer Vision, 2006:69(3), 335-351.

11. Cremers, D.. Nolinear Dynamical Shape Priors for Level Set Segmentation. CVPR, 2007.

12. R. P. Grzeszczuk and D. N. Levin, "Brownian strings: Segmented images with stochastically deformable contours," IEEE Trans. Pattern Anal. Mach. Intell., vol. 19, no. 10, pp. 1100-1114, Oct. 1997.

13. A. Cagnoni, A. Dobrzeniecki, R. Poli, and J. Yanch, "Genetic algorithmbased interactive segmentation of 3D medical images," Image Vis. Comput., vol. 17, no. 12, pp. 881-895, 1999.

14. L. Ballerini, "Genetic snakes for medical images segmentation," in Evolutionary Image Analysis, Signal Processing and Telecommunications, R. Poli, Ed. London, U.K.: Springer-Verlag, 1999, pp. 59-73.

15. M. Mignotte and J. Meunier, "Deformable template and distribution mixture-based data modeling for the endocardial contour tracking in an echographic sequence," in Proc. IEEE Comput. Soc. Conf. Comput. Vis. Pattern Recognit., 1999, vol. 1, pp. 225-230.

16. J. Montagnat and H. Delingette, "Globally constrained deformablemodels for 3Dobject reconstruction," Signal Process., vol. 71, no. 2, pp. 173-186, 1998.

17. J. Besag, "On the statistical analysis of dirty picture," J. Roy. Statist. Soc., vol. B-48, pp. 259-302, 1986.

18. M. Mignotte, C. Collect, P. Perez, and P. Bouthemy, "Unsupervised Markovian segmentation of sonar images," in Proc. Int. Conf. Acoust. Speech Signal Proc-ess., 1997, vol. 4, pp. 2781-2785.

19. B. Braathen, P. Masson, and W. Pieczynski, "Global and local methods of unsupervised Bayesian segmenta-tion of images," Graph. Vis., vol. 1, pp. 39-52, 1993.
20. Lifeng He, Yuyan Chao and Kenji Suzuki. A Run-Based Two-Scan Labeling Algo-rithm, IEEE transactions on Image Processing, 2008, 17(5): 749-756. 